\title{
Prospective 100 first results of immediate scoliosis correction with the new lyon brace: ARTbrace
}

\author{
Jean Claude de Mauroy ${ }^{1 *}$, Sophie Pourret ${ }^{2}$ \\ From 11th International Conference on Conservative Management of Spinal Deformities - SOSORT 2014 \\ Annual Meeting \\ Wiesbaden, Germany. 8-10 May 2014
}

\section{Background}

The immediate reducibility of the Cobb angle in brace is the fundamental parameter of success of non-surgical orthopedic treatment of scoliosis.

\section{Aim}

The objective of this work is to present the first results of the new Lyon brace (Asymmetrical Rigid Torsion brace) with immediate realization of the brace without plaster cast.

\section{Design}

The new Lyon brace is constructed with two asymmetrical lateral polycarbonate pieces connected posteriorly at the midline by a vertical incurved bar. The brace reproduce a twisted column in the opposite direction of scoliosis. The shape is obtained by superposition of three segmental electronic moldings which will be superimposed by a new software: OrtenShape.

\section{Results}

The results of a prospective series of the first 100 consecutive patients were studied using EOS X-ray and compared with results obtained by other braces.

Radiologically, in the frontal plane, the immediate in brace reduction is on average (0.72) $(0.63+-0.19$ for thoracic curves \& $0.76+-0.26$ for lumbar curves. These results can be classified:

- Depending on the type of curvature:

thoracolumbar (0.98),

lumbar (0.71),

double major $(0.67)$

lumbar (0.67),

thoracic $(0.64)$
- According to the criteria of the SRS (40 cases):

thoracic curves (0.66),

lumbar (0.83)

- According to the initial angulation:

$20-29^{\circ}=(0.80)$

$30-39^{\circ}=(0.65)$

$>40^{\circ}=(0.45)$

In 51 cases with initial kyphosis $<30^{\circ}$, improving the flat back is $9.25^{\circ}$ from $19^{\circ}$ to $28.25^{\circ}$.

Clinically, the push-up effect is : $1.64 \mathrm{~cm}$.

After at least 1 month of continuous wearing, for thoracic rib hump improvement is (0.50) and for lumbar (0.85).

The improvement is $40 \%$ compared to the old plaster cast and Lyon brace and 60\% compared to the Chêneau-Münster brace.

All radiological and clinical parameters improved significantly.

\section{Conclusion}

The new Lyon brace (ARTbrace) allows a better immediate in-brace reduction than the old Lyon brace. without need for a preliminary plaster cast.

\section{Authors' details}

${ }^{1}$ Clinique du Parc, Lyon, France. ${ }^{2}$ Lecante, Lyon, France.

Published: 4 December 2014

doi:10.1186/1748-7161-9-S1-035

Cite this article as: de Mauroy and Pourret: Prospective 100 first results of immediate scoliosis correction with the new lyon brace: ARTbrace. Scoliosis 2014 9(Suppl 1):035. 